\title{
MASIVE OPEN ONLINE COURSE (MOOC): EXPERIENCIAS EN LA FORMACIÓN DE PROFESORES UNIVERSITARIOS
}

\author{
MASIVE OPEN ONLINE COURSE (MOOC): EXPERIÊNCIAS NA FORMACÃO DE \\ PROFESSORES UNIVERSITÁRIOS
}

MASIVE OPEN ONLINE COURSE (MOOC): EXPERIENCES IN UNIVERSITY TEACHER TRAINING

DOI: 10.22481/rbba.v1i02.7796

Fabrício Oliveira da Silva Universidade Estadual de Feira de Santana, Bahia, Brasil ORCID: https://orcid.org/0000-0002-7962-7222 Lattes: http://lattes.cnpq.br/9101271365317978 Endereço eletrônico: fosilva@uefs.br

Yarelis Karina Araque Vergara Universidade Estadual de Feira de Santana, Bahia, Brasil ORCID: http://orcid.org/0000-0003-1083-4087 Lattes: http://lattes.cnpq.br/3769400811056185 Endereço eletrônico: yareliskarina@gmail.com

\section{RESUMEN}

El presente artículo tiene como objetivo identificar las experiencias educativas MOOC en la formación docente universitaria, a través de publicaciones académicas halladas en la web. Este estudio se realizó con la intención de saber qué resultados se han obtenido con esta modalidad educativa en la formación de profesores universitarios, frente a la creciente masificación de cursos virtuales experimentada en los últimos 
años. Por tanto, nuestra metodología de trabajo consistió en la identificación y uso de descriptores claves con operadores booleanos en el buscador Google Académico y en la base de datos SciELO, para direccionar la búsqueda de artículos relacionados con nuestra temática para su posterior selección, de acuerdo a criterios de inclusión y exclusión propuestos, lo que nos permitió construir un estado de arte sobre la temática. Así, fueron identificados varios tipos de MOOC, con diversas propuestas de participación y comunicación en la comunidad educativa, presentando muchos de ellos disimilitudes entre los objetivos, las metodologías y los resultados encontrados. De esta manera, concluimos que esta modalidad es una herramienta poderosa que propicia un canal valioso de intercambio de contenidos y saberes, a pesar de las debilidades que presenta el modelo en su proceso de implementación.

Palabras clave: MOOC; Formación de profesores; Relatos de experiencias; TIC.

\title{
RESUMO
}

Este artigo tem como objetivo identificar as experiências educacionais do MOOC na formação de professores universitários por meio de publicações acadêmicas baseadas na Web. Este estudo é realizado com o intuito de conhecer quais resultados foram obtidos com essa modalidade educacional na formação de professores universitários, dada a crescente massa de cursos virtuais vivenciados nos últimos anos. Portanto, uma metodologia de trabalho que consiste na identificação e uso de descritores-chave com operadores booleanos no mecanismo de pesquisa do Google Scholar e no banco de dados SciELO, para direcionar a pesquisa de artigos relacionados a uma seleção temática para uma seleção maior, de acordo com os critérios de inclusão e exclusão propostos, que nos permitiram construir um estado da arte sobre o assunto. Portanto, vários tipos de MOOCs foram identificados, com várias propostas de participação e comunicação na comunidade educacional, apresentando muitas das diferenças entre os objetivos, as metodologias e os resultados encontrados. Dessa forma, concluímos que essa modalidade é uma ferramenta poderosa que fornece um canal valioso para a troca de conteúdo e conhecimento, apesar das fragilidades do modelo em seu processo de implementação.

Palavras chave: MOOC; Formação de professores; Relatos de experiências; TIC.

\begin{abstract}
This article aims to identify the educational experiences of MOOC in the training of university teachers through web-based academic publications. This study is carried out in order to know what results were obtained with this educational modality in the training of university teachers, given the growing Masive Open Online Course experienced in recent years. Therefore, a working
\end{abstract}


methodology that identifies and uses key descriptors with Boolean operators in the Google Scholar search engine and SciELO database to direct the search for articles related to a thematic selection to a larger selection of according to the proposed inclusion and exclusion criteria, which allowed us to build a state of the art on the subject. Therefore, several types of MOOCs were identified, with several proposals for participation and communication in the educational community, presenting many of the differences between the objectives, the methodologies and the results found. Thus, we conclude that this modality is a powerful tool that provides a valuable channel for the exchange of content and knowledge, despite the weaknesses of the model in its implementation process.

Keywords: MOOC; Teacher Training; Experience stories; ICT.

\section{INTRODUCCIÓN}

Las Tecnologías de la Información y la Comunicación (TIC), son el conjunto de tecnologías desarrolladas para gestionar, almacenar y recuperar información, enviarla de un lugar a otro, tornar la comunicación más dinámica y rápida, abrir nuevas perspectivas, entre muchas otras posibilidades. Tienen un potencial enorme para apoyar el proceso de aprendizaje de los estudiantes, porque reducen principalmente obstáculos como distancia y tiempo lo que torna más dinámico el proceso. Sin embargo, por si solas no son efectivas, pues requieren ser aplicadas y pensadas con una intencionalidad, para que el proceso de enseñanza se dé significativamente, además deben encontrar un balance entre el número de herramientas, los recursos utilizados, las actividades y los aprendizajes que se desean promover en los discentes.

Actualmente, las TIC son fundamentales en las aulas, esto lo podemos percibir en la implementación y uso de: las computadoras con conexión a internet, los proyectores, los videos, etc. No obstante, el uso de la tecnología en el salón de clase no es algo nuevo, pues se ha manifestado en el proceso de enseñanza durante décadas. Ejemplo de esto, es el tradicional pizarrón ${ }^{\mathrm{i}}$ (GALAVIZ PEÑA, 2014). Ahora, las TIC aplicadas en la formación de profesores, las encontramos en una nueva modalidad que está siendo implementada en diferentes universidades y que se ha tornado hoy en día, una tendencia mundial, que consiste en apoyar los aprendizajes de los estudiantes, matriculados o no dentro de una casa de estudio, mediante iniciativas como cursos online en los que pueden acceder desde la internet con relativa facilidad, de forma gratuita y seguir un ritmo individualizado de aprendizaje. Ejemplo en España las Universidades de Cantabria (UC) y Oviedo (UO).

Revista RBBA Revista Binacional Brasil Argentina 

PROFESORES UNIVERSITARIOS

En el año 2011, los profesores Sebastian Thrun, profesor de la Universidad de Stanford y Peter Norvig, director de investigación de Google crearon el primer curso exitoso sobre inteligencia artificial bajo la modalidad Massive Online Open Courses (Cursos online masivos y abiertos) o MOOC por su acrónimo en inglés, en el que se matricularon 160.000 personas. El término MOOC ya había sido anteriormente acuñado por Dave Cormier para referirse al curso Conectivismo y Aprendizaje Conectado (CCK08), dirigido en 2008 por los profesores de la Universidad de Manitoba, George Siemens y Stehpen Downes en Canadá.

Hoy en día, el número de MOOC disponibles en la Red se encuentra en franco crecimiento, porque además de ser gratuito, abierto y accesible para cualquier usuario, son considerado en la literatura divulgativa y científica como una revolución con un gran potencial en el mundo educativo y formativo (VÁZQUEZ CANO; LÓPEZ MENESES, 2014).

Los MOOC han acaparado un interés mundial debido a su gran potencial para ofrecer una formación gratuita, de calidad y accesible a cualquier persona independientemente de su procedencia, su formación previa y sin la necesidad de pagar por su matrícula. Sin embargo, en la actualidad se desconoce cuáles son los resultados y alcances de los aprendizajes en los estudiantes, los elementos del diseño instruccional, la calidad de los materiales y las principales dificultades que se afrontan cuando se diseñan este tipo de cursos.

En este sentido, nos preguntamos ¿cuáles han sido los resultados obtenidos de las experiencias educativas con MOOC en la formación de profesores universitarios en los cuatro últimos años? Para responder esta pregunta (nos) hemos propuesto identificar estas experiencias en la formación docente universitaria, a través de publicaciones académicas halladas en la web, desde enero del 2015 hasta agosto del 2019.

\section{METODOLOGIA}

La estrategia metodológica adoptada se ha centrado en las características propias de un estado de arte (ROMANOWSKI; TEODORA ENS, 2006) donde se señala que un levantamiento es una revisión del conocimiento producido sobre el tema, es un paso indispensable para desencadenar un proceso de análisis cualitativo de estudios producidos en diferentes áreas del conocimiento. Posibilitando así una contribución para identificar aportes significativos en la constitución de un área del conocimiento. 

PROFESORES UNIVERSITARIOS

Consecuentemente, la búsqueda fue realizada en la base de datos SciELO y en el buscador google académico, ambos con reconocido prestigio en la comunidad académica en Ciencias Sociales, como también en las Ciencias de la Educación. Así, la búsqueda de los datos se hizo en plataformas, las cuales describimos a seguir, describiendo su naturaleza.

Con relación al Google Académico, este es el buscador de Google especializado en literatura científica o académica, que permite localizar información de forma sencilla. Permite buscar en varias fuentes al mismo tiempo, localizar documentos completos, rastrear artículos por autor o dar un artículo que aparece en una publicación en concreto.

En el caso de Scielo, esta es la plataforma identificada como la Scientific Electronic Library Online (SciELO) que es una biblioteca electrónica que cubre una colección seleccionada de revistas científicas brasileñas. La plataforma es el resultado de un proyecto de investigación de la Fundación de Apoyo a la Investigación del Estado de São Paulo (FAPESP), en asociación con el Centro Latinoamericano y del Caribe de Información en Ciencias de la Salud (BIREME).

Para el desarrollo de la presente investigación, se definieron los descriptores para direccionar la búsqueda de artículos relacionados con la temática. El establecimiento de palabras se realizó en dos idiomas: español y luego traducido al portugués. En español se utilizaron las palabras: "relatos experiencias", "MOOC”, “educación superior”, y “educación universitaria", en portugués fueron: "relatos de experiência", "MOOC", "ensino superior", "ensino universitário".

Posteriormente, utilizando los operadores booleanos "OR" y "AND" se formuló la siguiente ecuación de búsqueda aplicada en Google académico: en español: "relatos experiencias AND MOOC AND educación superior OR educación universitaria”. En esa primera búsqueda se consiguieron 1902 publicaciones de las cuales 1050 estaban en español y 852 en idioma portugués. En SciELO se realizó la primera búsqueda utilizando las mismas palabras que en Google académico, pero no se consiguió ningún trabajo; entonces se procedió a realizar búsqueda con la palabra MOOC filtrando la búsqueda en idiomas español y portugués, encontrando 32 artículos.

Luego en una segunda fase se realizó una selección incluyendo solo artículos de revistas, publicados entre enero de 2015 y agosto de 2019, publicados en abierto y disponible para su consulta. Fueron excluidos en esta etapa actas de congresos, libros (capítulos) y otros tipos de 

PROFESORES UNIVERSITARIOS

publicaciones o artículos con acceso restringido, de los cuales resultaron seleccionados 44 artículos en Google académico y 32 en SciELO.

En la tercera fase, se seleccionaron los artículos relacionados con MOOC en la formación de profesores, en la que se relata la experiencia en diversos cursos bajo esta modalidad. En esta etapa fueron excluidos de la selección los artículos duplicados que fueron hallados en ambas bases de datos. Seleccionando un total de 8 artículos para realizar el análisis final de los cuales 7 fueron de Google académico y 2 de SciELO.

Los criterios de inclusión y exclusión, para esta revisión se presentan en la tabla 1.

Tabla 1. Criterios de inclusión y exclusión.

\begin{tabular}{|l|l|}
\hline \multicolumn{1}{|c|}{ Critérios de inclusión } & \multicolumn{1}{|c|}{ Critérios de exclusión } \\
\hline a. Artículos de revista. & $\begin{array}{l}\text { a. Actas de congresos, capítulos de libro, } \\
\text { libros u otro tipo de publicaciones. }\end{array}$ \\
\hline $\begin{array}{l}\text { b. Publicaciones entre enero de } 2015 \text { hasta } \\
\text { agosto de } 2019 .\end{array}$ & b. Acceso restringido a la publicación. \\
\hline $\begin{array}{l}\text { c. Publicado en abierto y disponible para su } \\
\text { consulta. }\end{array}$ & c. Artículos teóricos. \\
\hline d. MOOC en la formación de profesores. & d. Artículos duplicados. \\
\hline
\end{tabular}

El procedimiento que se ha seguido en la elaboración del estado del arte constó de tres fases diferenciadas a partir de la introducción de una serie de criterios de inclusión (tabla 1). La primera fase correspondió a la búsqueda inicial donde fueron implementados los operadores boléanos (OR, AND) para la búsqueda en el buscador de Google académico y en la plataforma SciELO.

Posteriormente, en una segunda fase, los resultados fueron filtrados de acuerdo a los criterios (a), (b) y (c) de inclusión y (a) y (b) de exclusión. Finalmente, en la tercera fase se examinaron minuciosamente el título y resumen de los resultados obtenidos, siendo seleccionados aquellos acordes a los criterios (d) de inclusión y (c) y (d) de exclusión que se encuentra esquemáticamente presentados en la figura 1. 
Figura 1. Fase de la elaboración del estado de arte.

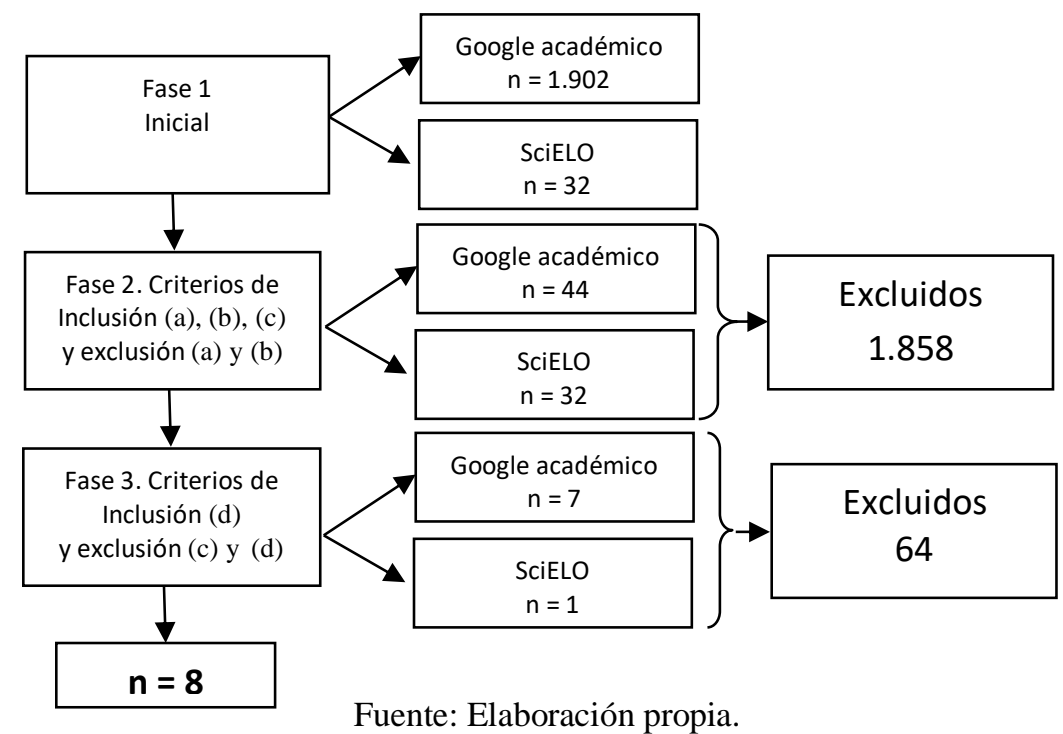

La muestra final de artículos que se consideraron en el presente estado del arte quedo constituido por 8 artículos, que relatan las experiencias con MOOC en la formación de profesores en diferentes latitudes.

Se han identificado 5 variables de interés para el análisis del efecto del MOOC en la formación de profesores. Las variables objeto de estudio se han dividido según su tipología en (1) Tipo de MOOC, (2) Criterios diferenciadores "abierto, gratuito y fomento de comunidades de aprendizaje", (3) Actividades propuestas. Y aspectos metodológicos como: (4) diseño metodológico, y (5) calidad pedagógica de los MOOC.

\section{RESULTADOS}

En la selección de artículos para desarrollar el presente estado de arte hemos identificado 8 artículos que se proponen realizar un análisis de cursos MOOC en la formación de profesores. La tabla 2 presenta los aspectos más relevantes de cada uno de los artículos, como: autor, año, objetivo, metodología, resultados y conclusiones.

En cada uno de los trabajos de investigación nos preocupamos por identificar el tipo de MOOC del que se trata, los criterios diferenciadores de MOOC, el diseño metodológico, las actividades propuestas y el modelo pedagógico. 

PROFESORES UNIVERSITARIOS

Los resultados revelaron en lo referente al tipo de MOOC, que se identificaron dos tipos (xMOOC y cMOOC) con diversas propuestas de participación y comunicación entre los miembros de la comunidad educativa. Muchos de ellos presentan con disimilitud entre los objetivos, las metodologías y los resultados encontrados. Sin embargo, se logró identificar que el tipo de MOOC que predomina es el de tipo xMOOC que son un tipo de curso tradicional basado en modelos comunicativos conductistas, que surgen para reproducir una pedagogía tradicional.

En cuanto a los criterios diferenciadores de MOOC, tales como abierto, gratuito y fomento de una comunidad de aprendizaje, en las experiencias con MOOC consultadas, se puedó observar que la mayoría de los cursos cumplen las características de ser abierto y gratuitos, pero tienen dificultades en la formación de comunidades virtuales de aprendizaje.

Por otra parte, se observó que la dinámica del curso no genera comunidades virtuales de aprendizaje, ni promueven la comunicación entre los participantes, lo que en cierto modo redunda en que la comunicación entre docentes y participantes sea también muy reducida.

En lo que respecta a los diseños metodológicos de los MOOC, son cursos que en su mayoría ofrecen un modelo basado en clases magistrales, fragmentadas en presentaciones en formato audiovisual y el nivel de competencia que se les exige a los participantes se basa en el aprendizaje memorístico. Bajo la filosofía MOOC, el diseño de estos cursos requiere la participación activa de los estudiantes en la construcción de los materiales, y no solo partir de un buen contenido elaborado por profesores universitarios con gran experiencia o pericia técnica.

Una de las debilidades encontradas y que es recurrentemente mencionada en las experiencias con MOOC consultadas, fue la baja creación de comunidades de aprendizaje, y que las actividades propuestas no transcienden la asimilación de contenidos y posterior evaluación, a pesar de ser cursos que se encuentran alojados en plataformas de reconocido prestigio internacional.

Por otra parte, existe entre los docentes el temor que el acceso a MOOC promueva su desvinculación con el campo laboral, por sustitución del docente por esta herramienta, lo que puede representar una limitante en la implementación efectivas de este tipo ambientes virtuales de aprendizaje, pues los docentes no se ven como una parte importante del diseño de experiencias educativas con MOOC. 

PROFESORES UNIVERSITARIOS

Respecto a las actividades propuestas, como ya se mencionó los cursos ofrecen un modelo basado en clases magistrales, fragmentadas en presentaciones, en formato audiovisual. En algunas experiencias, se pudo observar que los principales cuestionamientos fueron: excesiva facilidad que se da en algunos de estos cursos para su superación, existencia de actividades muy pobres desde un punto de vista pedagógico, y la preponderancia del cuestionario como actividad a realizar. En menor medida, el uso limitado de algunas herramientas de comunicación y la ausencia de adaptaciones de acceso a la información para el caso de estudiantes con algún tipo de dificultad.

El Modelo pedagógico de los MOOC parece indicar por lo menos en los artículos consultados, que lo más importante en esta iniciativa es su modelo pedagógico abierto y colaborativo fundamentado en la teoría conectivista (RODRÍGUEZ; MOLERO, 2008), y su modelo de aprendizaje que se genera, gracias al intercambio de información propicia para la participación en un modelo de construcción colectiva, que organiza a las comunidades discursivas para que promuevan el conocimiento en colectivo. Sin embargo, la calidad pedagógica de los MOOC es percibida como deficiente, debido a la simplificación de los procesos de enseñanza, derivada de una comprensión muy limitada de la función del profesorado y de su papel en la potenciación de los aprendizajes.

Tabla 2. Experiencias con MOOC.

\begin{tabular}{|c|c|c|c|c|}
\hline Autor (año) & Objetivo & Metodología & Resultados & Conclusiones \\
\hline $\begin{array}{l}\text { (CALVO } \\
\text { SALVADOR; } \\
\text { BRAGA } \\
\text { BLANCO, 2019) }\end{array}$ & $\begin{array}{l}\text { Realizar un análisis } \\
\text { pedagógico de cursos } \\
\text { MOOC ofertados en } \\
\text { castellano en } \\
\text { Universidades de } \\
\text { Cantabria y Oviedo } \\
\text { en España. }\end{array}$ & $\begin{array}{l}\text { Investigación de } \\
\text { corte cualitativo, se } \\
\text { utilizaron técnicas de } \\
\text { recogida de datos } \\
\text { como las } \\
\text { observaciones, } \\
\text { diarios de campo, } \\
\text { grupos de discusión } \\
\text { y entrevistas. }\end{array}$ & $\begin{array}{l}\text { Resalta la } \\
\text { importancia que ha } \\
\text { tenido el trabajo de } \\
\text { análisis realizado } \\
\text { con los estudiantes } \\
\text { en el marco de } \\
\text { varias asignaturas de } \\
\text { las titulaciones de } \\
\text { educación, tanto por } \\
\text { los resultados } \\
\text { obtenidos como por } \\
\text { el proceso de trabajo } \\
\text { desarrollado. }\end{array}$ & $\begin{array}{l}\text { Es necesario acercar } \\
\text { el fenómeno de los } \\
\text { MOOC a los estudios } \\
\text { educativos, } \\
\text { ampliando el } \\
\text { horizonte de los } \\
\text { mismos con el } \\
\text { abordaje de nuevos } \\
\text { fenómenos sociales y } \\
\text { educativos, así como } \\
\text { realizar un análisis } \\
\text { crítico de este tipo de } \\
\text { formación on-line } \\
\text { que no solamente } \\
\text { tenga en cuenta } \\
\text { factores tecnológicos } \\
\text { o económicos, sino } \\
\text { también elementos } \\
\text { pedagógicos. }\end{array}$ \\
\hline $\begin{array}{l}\text { (MOREIRA } \\
\text { TEIXEIRA et al., } \\
\text { 2018) }\end{array}$ & $\begin{array}{l}\text { Analisar em que } \\
\text { medida a aplicação e } \\
\text { aprofundamento } \\
\text { socioconstrutivistas }\end{array}$ & $\begin{array}{l}\text { A metodologia do } \\
\text { estudo assenta no } \\
\text { modelo design-based } \\
\text { research. Esta }\end{array}$ & $\begin{array}{l}\text { Considerando que a } \\
\text { aprendizagem se } \\
\text { desenvolve através } \\
\text { da interação com os }\end{array}$ & $\begin{array}{l}\text { Evidencia a } \\
\text { contribuição deste } \\
\text { modelo pedagógico } \\
\text { inovador para a }\end{array}$ \\
\hline
\end{tabular}




\begin{tabular}{|c|c|c|c|c|}
\hline & $\begin{array}{l}\text { que combinam a } \\
\text { dimensão da } \\
\text { autoaprendizagem } \\
\text { com a dimensão } \\
\text { social no MOOC de } \\
\text { Competências } \\
\text { Digitais para } \\
\text { Professores promove } \\
\text { a apropriação de } \\
\text { conhecimento e } \\
\text { partilha de } \\
\text { experiências, bem } \\
\text { como o } \\
\text { desenvolvimento de } \\
\text { competências } \\
\text { digitais. }\end{array}$ & $\begin{array}{l}\text { metodologia } \\
\text { apresenta } \\
\text { potencialidades } \\
\text { quando se pretende } \\
\text { criar e expandir } \\
\text { conhecimento } \\
\text { relacionado com o } \\
\text { desenvolvimento, a } \\
\text { implementação e a } \\
\text { sustentação de } \\
\text { ambientes de } \\
\text { aprendizagem } \\
\text { inovadores. }\end{array}$ & $\begin{array}{l}\text { materiais, os } \\
\text { facilitadores e entre } \\
\text { os participantes, } \\
\text { procedeu-se à } \\
\text { análise das } \\
\text { interações ocorridas } \\
\text { no espaço virtual; } \\
\text { numa segunda fase, } \\
\text { realizou-se uma } \\
\text { análise de conteúdo } \\
\text { das diferentes } \\
\text { produções dos } \\
\text { participantes. }\end{array}$ & $\begin{array}{l}\text { apropriação de novos } \\
\text { conhecimentos e a } \\
\text { reflexão sobre as } \\
\text { práticas, destacando } \\
\text { a possibilidade } \\
\text { destes cursos se } \\
\text { constituírem como } \\
\text { um instrumento } \\
\text { poderoso na } \\
\text { formação de } \\
\text { professores e outros } \\
\text { profissionais, } \\
\text { proporcionando } \\
\text { pontos de reflexão } \\
\text { sobre as novas } \\
\text { abordagens } \\
\text { pedagógicas que } \\
\text { emergem a partir dos } \\
\text { MOOCs.. }\end{array}$ \\
\hline $\begin{array}{l}\text { (MANOTAS } \\
\text { SALCEDO; } \\
\text { PÉREZ } \\
\text { RODRÍGUEZ; } \\
\text { CONTRERAS } \\
\text { PULIDO, 2018) }\end{array}$ & $\begin{array}{l}\text { Analizar un grupo de } \\
\text { vídeo-lecciones } \\
\text { contenidas en } \\
\text { MOOC enfocados en } \\
\text { la formación } \\
\text { pedagógica de } \\
\text { docentes de } \\
\text { Educación Superior } \\
\text { en España. }\end{array}$ & $\begin{array}{l}\text { Se realizó el análisis } \\
\text { de contenido de } 104 \\
\text { vídeo-lección en } 3 \\
\text { cursos publicados en } \\
\text { la plataforma en } \\
\text { español Miriada X. } \\
\text { El proceso tuvo dos } \\
\text { fases, una, en la que } \\
\text { se diseñó e } \\
\text { implementó un } \\
\text { instrumento para } \\
\text { caracterizar el inicio, } \\
\text { el desarrollo y el } \\
\text { cierre de las vídeo- } \\
\text { lecciones como } \\
\text { unidad de } \\
\text { comunicación, y } \\
\text { otra, que consistió en } \\
\text { la organización, } \\
\text { transcripción y } \\
\text { codificación de las } \\
\text { vídeo- lecciones } \\
\text { mediante el criterio } \\
\text { de cronología, que } \\
\text { permitió determinar } \\
\text { tendencias y } \\
\text { patrones. }\end{array}$ & $\begin{array}{l}\text { Se evidenció un uso } \\
\text { básico de } \\
\text { herramientas } \\
\text { audiovisuales y la } \\
\text { tendencia a diseñar } \\
\text { clases netamente } \\
\text { expositivas } \\
\text { detonando ausencia } \\
\text { de elementos de } \\
\text { storytelling. }\end{array}$ & $\begin{array}{l}\text { Los resultados son } \\
\text { relevantes para el } \\
\text { diseño instruccional } \\
\text { de recursos } \\
\text { audiovisuales para } \\
\text { cursos masivos y } \\
\text { para configurar } \\
\text { propuestas creativas } \\
\text { en el diseño de } \\
\text { ambientes virtuales } \\
\text { de aprendizaje, } \\
\text { especialmente } \\
\text { enfocados en la } \\
\text { formación docente. } \\
\text { Al realizar un curso } \\
\text { en formación } \\
\text { pedagógica, los } \\
\text { docentes esperarían } \\
\text { poder conocer y } \\
\text { aprender sobre } \\
\text { modelos y estrategias } \\
\text { de aprendizaje activo } \\
\text { para incorporar en } \\
\text { sus aulas. }\end{array}$ \\
\hline $\begin{array}{l}\text { (GIL QUINTANA, } \\
\text { 2017) }\end{array}$ & $\begin{array}{l}\text { Descubrir la función } \\
\text { del alumnado y el } \\
\text { profesorado en la } \\
\text { pedagogía } \\
\text { interactiva de los } \\
\text { MOOC. }\end{array}$ & $\begin{array}{l}\text { Análisis se } \\
\text { complementa } \\
\text { con la investigación } \\
\text { cualitativa efectuada } \\
\text { a través de un } \\
\text { proceso donde } \\
\text { pretendemos } \\
\text { indagar sobre } \\
\text { "motivaciones, } \\
\text { representaciones y, } \\
\text { en general, aquellos }\end{array}$ & $\begin{array}{l}\text { La plataforma del } \\
\text { MOOC tiene una } \\
\text { vital importancia en } \\
\text { su desarrollo, } \\
\text { tipología de cursos } \\
\text { elegida por los } \\
\text { equipos docentes del } \\
\text { Proyecto Europeo } \\
\text { ECO para su } \\
\text { plataforma. Estos } \\
\text { espacios se }\end{array}$ & $\begin{array}{l}\text { Los MOOC } \\
\text { responden a una } \\
\text { nueva realidad de } \\
\text { formación virtual } \\
\text { que se está } \\
\text { potenciando en } \\
\text { España desde } 2014 \text { a } \\
\text { través del Proyecto } \\
\text { ECO. El crecimiento } \\
\text { se relaciona con } \\
\text { factores que van más }\end{array}$ \\
\hline
\end{tabular}




\begin{tabular}{|c|c|c|c|c|}
\hline & & $\begin{array}{l}\text { objetivos cuya } \\
\text { principal manera de } \\
\text { manifestarse es a } \\
\text { través del discurso } \\
\text { de la gente" }\end{array}$ & $\begin{array}{l}\text { abren al plano social } \\
\text { y difunden los } \\
\text { planteamientos e } \\
\text { ideas que, dentro del } \\
\text { proceso formativo } \\
\text { se generan, } \\
\text { provocando el } \\
\text { desarrollo de un } \\
\text { modelo } \\
\text { comunicativo } \\
\text { horizontal y } \\
\text { bidireccional. }\end{array}$ & $\begin{array}{l}\text { allá de las } \\
\text { posibilidades } \\
\text { pedagógicas que } \\
\text { estas propuestas } \\
\text { ofrecen. } \\
\text { El papel del } \\
\text { profesorado es } \\
\text { fundamental para } \\
\text { potencializar la } \\
\text { interactividad y la } \\
\text { participación del } \\
\text { alumnado en estos } \\
\text { espacios digitales, en } \\
\text { la comunidad virtual } \\
\text { de aprendizaje se ha } \\
\text { generado la } \\
\text { participación de la } \\
\text { ciudadanía en la } \\
\text { construcción } \\
\text { colectiva del } \\
\text { conocimiento. }\end{array}$ \\
\hline $\begin{array}{l}\text { (GIL QUINTANA, } \\
\text { 2015) }\end{array}$ & $\begin{array}{l}\text { Analizar las ventajas } \\
\text { e inconvenientes del } \\
\text { MOOC como } \\
\text { modelo } \\
\text { educomunicativo de } \\
\text { formación. } \\
\text { Observar el papel de } \\
\text { las redes sociales } \\
\text { dentro de la } \\
\text { metodología } \\
\text { educomunicativa de } \\
\text { los MOOC en } \\
\text { España. }\end{array}$ & $\begin{array}{l}\text { Investigación } \\
\text { cualitativa, } \\
\text { utilizando como } \\
\text { instrumento la } \\
\text { entrevista a una } \\
\text { muestra intencional } \\
\text { de profesores y } \\
\text { estudiantes de la } \\
\text { comunidad virtual de } \\
\text { aprendizaje en la que } \\
\text { se desarrolló el } \\
\text { curso. }\end{array}$ & $\begin{array}{l}\text { El grado de } \\
\text { satisfacción de } \\
\text { profesores y } \\
\text { estudiantes es } \\
\text { grande, en } \\
\text { comparación con el } \\
\text { número de } \\
\text { desventajas o } \\
\text { inconvenientes que } \\
\text { se señalaron. } \\
\text { Se destaca que la } \\
\text { apertura, la } \\
\text { gratuidad, el } \\
\text { carácter masivo y } \\
\text { online, son ventajas } \\
\text { de este tipo de } \\
\text { formación que } \\
\text { además cuenta con } \\
\text { el respaldo de } \\
\text { instituciones } \\
\text { universitarias } \\
\text { abiertas al mundo } \\
\text { global. } \\
\text { Entre los } \\
\text { inconvenientes se } \\
\text { encuentra la alta } \\
\text { tasa de abandono, el } \\
\text { coste de los } \\
\text { servidores y la } \\
\text { incompetencia de } \\
\text { las empresas que } \\
\text { desarrollan los } \\
\text { espacios digitales } \\
\text { para fomentar } \\
\text { herramientas } \\
\text { comunicativas que }\end{array}$ & $\begin{array}{l}\text { La implantación en } \\
\text { estos cursos será una } \\
\text { realidad cuando se } \\
\text { ofrezca un itinerario } \\
\text { abierto con la } \\
\text { posibilidad para } \\
\text { participar en el } \\
\text { diseño de los } \\
\text { contenidos y de } \\
\text { proponer diferentes } \\
\text { cuestiones de debate, } \\
\text { partiendo de su } \\
\text { propio interés de } \\
\text { estudiantes y } \\
\text { profesores. } \\
\text { Los docentes temen } \\
\text { que el acceso } \\
\text { promueva su } \\
\text { desvinculación con } \\
\text { otros contratos para } \\
\text { el desarrollo de su } \\
\text { propio trabajo y su } \\
\text { presencia en } \\
\text { asignaturas de una } \\
\text { determinada } \\
\text { titulación. Sin } \\
\text { embargo, el papel } \\
\text { motivador del } \\
\text { docente, es un } \\
\text { elemento clave para } \\
\text { poder desarrollar } \\
\text { MOOC. }\end{array}$ \\
\hline
\end{tabular}




\begin{tabular}{|c|c|c|c|c|}
\hline & & & $\begin{array}{l}\text { favorezcan la } \\
\text { interacción. Y entre } \\
\text { los profesores } \\
\text { preocupación por la } \\
\text { desaparición o el } \\
\text { abaratamiento de } \\
\text { puestos de trabajos } \\
\text { en la universidad. }\end{array}$ & \\
\hline $\begin{array}{l}\text { (MALLAMANN; } \\
\text { NOBRE } \\
\text { FERREIRA, 2017) }\end{array}$ & $\begin{array}{l}\text { Reflexionar sobre a } \\
\text { contribuição e } \\
\text { organização didática } \\
\text { dos Recursos } \\
\text { Educacionais } \\
\text { Abertos (REA) e } \\
\text { MOOC na formação } \\
\text { universitária } \\
\text { mediada por } \\
\text { tecnologias } \\
\text { educacionais no } \\
\text { século XXI }\end{array}$ & $\begin{array}{l}\text { Investigação de tipo } \\
\text { Qualitativa, foi } \\
\text { analisado dados de } \\
\text { dois MOOC } \\
\text { internacionais que } \\
\text { versavam sobre } \\
\text { REA. Como } \\
\text { resultados da } \\
\text { participação ativa } \\
\text { nos cursos, foram } \\
\text { registrados planos de } \\
\text { cursos, materiais } \\
\text { didáticos, } \\
\text { metodologia de } \\
\text { avaliação, atividades } \\
\text { de aprendizagem, } \\
\text { interação entre } \\
\text { participantes e } \\
\text { partilha online. }\end{array}$ & $\begin{array}{l}\text { Evidenciaram-se } \\
\text { elementos didáticos } \\
\text { diversificados nos } \\
\text { MOOC com ênfase } \\
\text { em vídeos e } \\
\text { variação entre carga } \\
\text { horária, tipo e } \\
\text { número de } \\
\text { atividades semanais. } \\
\text { Por outro lado, foi } \\
\text { atribuída aos } \\
\text { próprios } \\
\text { participantes } \\
\text { autonomia para } \\
\text { definir as suas } \\
\text { próprias metas de } \\
\text { aprendizagem, } \\
\text { determinar a } \\
\text { natureza e a } \\
\text { extensão temporal } \\
\text { da sua participação } \\
\text { e também quais } \\
\text { seriam as } \\
\text { ferramentas que } \\
\text { usariam para criar } \\
\text { os artefatos que irão } \\
\text { constituir o principal } \\
\text { inventário } \\
\text { substantivo do curso } \\
\text { no final } \\
\text { (diversidade). }\end{array}$ & $\begin{array}{l}\text { É necessária uma } \\
\text { reflexão sobre as } \\
\text { estratégias didáticas } \\
\text { e a composição } \\
\text { congruente de } \\
\text { políticas de formação } \\
\text { universitária. Os } \\
\text { impactos de um } \\
\text { curso MOOC são } \\
\text { numerosos e as suas } \\
\text { consequências } \\
\text { afetam tanto os } \\
\text { participantes como } \\
\text { os professores e o } \\
\text { sistema educativo, } \\
\text { inclusive as políticas } \\
\text { de financiamento da } \\
\text { educação. } \\
\text { É necessário investir } \\
\text { em políticas } \\
\text { institucionais para } \\
\text { consolidar a } \\
\text { educação aberta. }\end{array}$ \\
\hline $\begin{array}{l}\text { (LACERDA } \\
\text { RABELLO; } \\
\text { AMARAL } \\
\text { TAVARES, 2017) }\end{array}$ & $\begin{array}{l}\text { Investigar através da } \\
\text { análise de dados de } \\
\text { linguagem, a } \\
\text { contribuição de um } \\
\text { curso on-line de } \\
\text { formação continuada } \\
\text { de professores com } \\
\text { vistas à integração } \\
\text { das tecnologias } \\
\text { digitais no ensino } \\
\text { superior. }\end{array}$ & $\begin{array}{l}\text { Um estudo de caso } \\
\text { de base etnográfica }\end{array}$ & $\begin{array}{l}\text { O artigo identifica } \\
\text { as contribuições da } \\
\text { metodologia e } \\
\text { desenho do curso } \\
\text { para o letramento } \\
\text { digital dos } \\
\text { professores e a } \\
\text { formação } \\
\text { continuada em } \\
\text { relação à integração } \\
\text { das tecnologias } \\
\text { digitais à prática } \\
\text { docente na } \\
\text { universidade. }\end{array}$ & $\begin{array}{l}\text { Os critérios e } \\
\text { princípios adotados } \\
\text { na elaboração do } \\
\text { curso de formação } \\
\text { continuada } \\
\text { mostraram-se } \\
\text { eficazes no sentido } \\
\text { de promover o } \\
\text { letramento digital } \\
\text { dos professores e a } \\
\text { apropriação crítica } \\
\text { de diferentes } \\
\text { tecnologias digitais } \\
\text { indicando mudanças } \\
\text { na prática docente. }\end{array}$ \\
\hline $\begin{array}{l}\text { (BENTET GIL et } \\
\text { al., 2018) }\end{array}$ & $\begin{array}{l}\text { Conocer la } \\
\text { percepción de los } \\
\text { participantes sobre el }\end{array}$ & $\begin{array}{l}\text { Estudio de corte } \\
\text { descriptivo, } \\
\text { sustentado en el }\end{array}$ & $\begin{array}{l}\text { La mayoría de los } \\
\text { sujetos contestaron } \\
\text { positivamente la }\end{array}$ & $\begin{array}{l}\text { Se percibe el MOOC } \\
\text { como una } \\
\text { herramienta }\end{array}$ \\
\hline
\end{tabular}




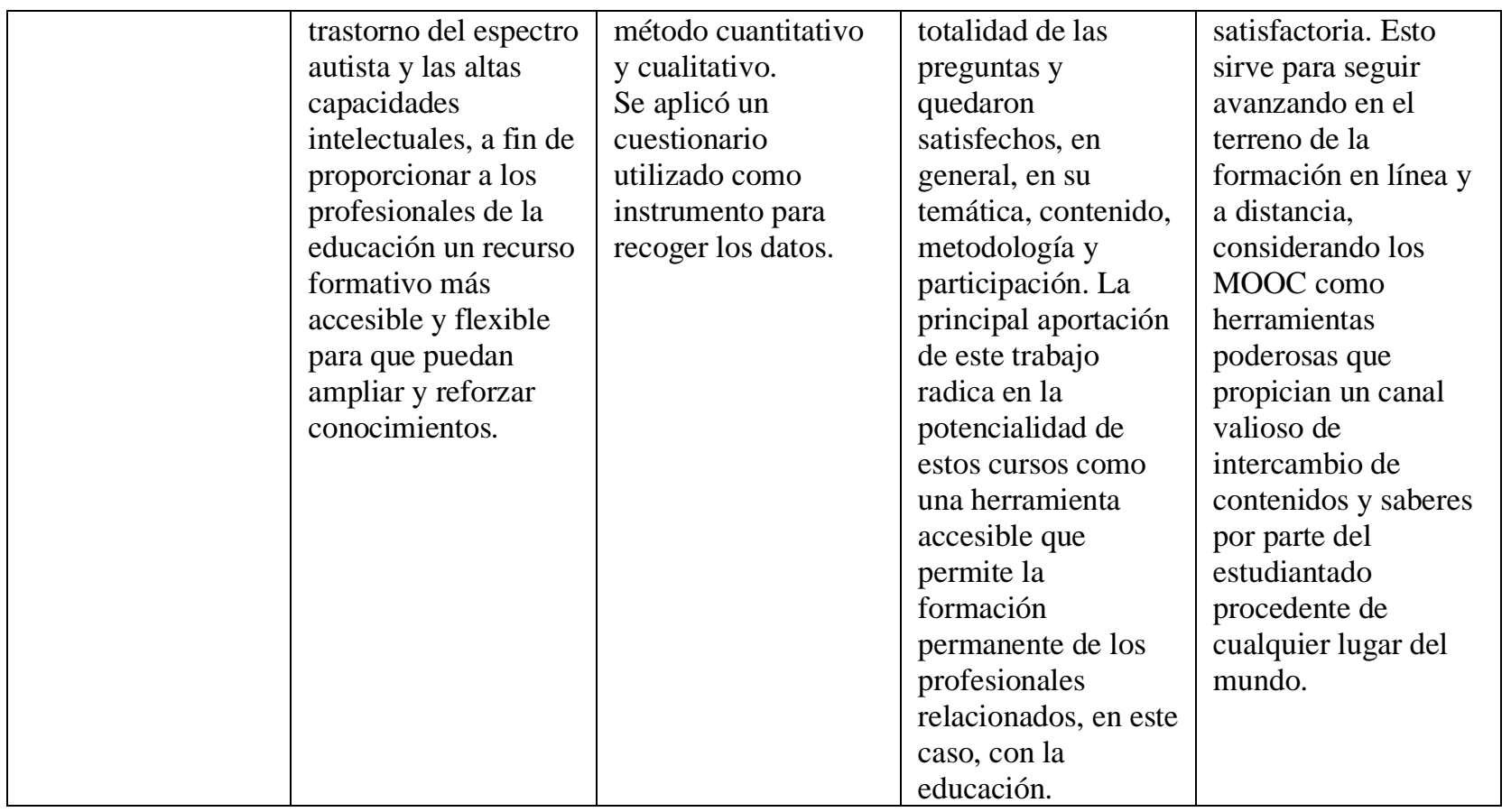

Fuente: Elaboración propia.

\section{CONCLUSIÓN}

La creación de comunidades virtuales de aprendizaje en las que se construye el conocimiento en colaboración, como los MOOC se han visto perjudicadas por el mal funcionamiento de la plataforma, deficiencias metodológicas y pedagógicas exhibidas en algunos cursos. El entorno digital enmarcado en este modelo de formación masivo y abierto, posibilita la creación de una especie de intelecto colectivo, con otras personas del mundo que se encuentran conectadas en la Red. Sin embargo, creemos que los problemas que hemos identificado dificultan la organización de los contenidos, e impiden el desarrollo de un modelo comunicativo multidireccional y horizontal.

En este sentido, coincidimos con los autores de los artículos analizados en reconocer que los MOOCs son herramientas poderosas que propician un canal valioso de intercambio de contenidos y saberes, pero aún quedan muchas interrogantes por responder tales como ¿Qué relaciones pedagógicas se establecen en estos entornos de aprendizaje? ¿Qué tipo de relaciones comunicativas son las más relevantes?, ¿Cuál es el criterio para valorar la calidad de los recursos que se ofrecen en estas plataformas? y ¿Cómo se puede ser compatible la masificación de estos cursos con atención a la diversidad e individualización de los aprendizajes?

En este sentido, a pesar de ser una herramienta interesante para mejorar la formación del profesorado, existen problemas de limitación sobre las acciones educativas que deberían ser 

PROFESORES UNIVERSITARIOS

problematizadas en el campo de la formación inicial del profesorado, que incluyen la relación entre el profesor y el alumno. Dichas relaciones deben constituirse a partir de experiencias reales del campo educativo, con una centralidad en los eventos de la profesión docente. Por lo tanto, los problemas de la vida escolar diaria y la realidad educativa deben estar presentes en los cursos de formación de docentes, un hecho que poco se puede hacer con este tipo de herramienta y modo formativo en el que la relación no se produce en el contexto real y situacional de la enseñanza. Esto implica reconocer que la formación del profesorado no solo puede limitarse a reflexiones sobre la profesión docente, sino sobre todo a la inserción dentro de la profesión misma. Los cursos de tipo MOOC se limitan a problematizar tales experiencias en el campo de la educación, pero no genera experiencias en el campo relacional y de la inserción en los eventos de la profesión, para lo cual el maestro necesita, en su formación, adaptarse a la vida cotidiana de la enseñanza que él, a través de los cursos MOOC refleja.

Sin embargo, los artículos analizados problematizan situaciones relevantes en la formación docente, especialmente cuando reflejan resultados que muestran la necesidad de la producción de estrategias de enseñanza, al abordar cuestiones curriculares a tener en cuenta; cuando enfatizan que la capacitación en cursos de tipo MOOC es autónoma. Es un elemento clave en el proceso de capacitación, cuando se enfocan en la naturaleza misma de los cursos en línea, ya que los mecanismos poderosos para pensar sobre la amplitud de la información que manipulan pueden generar un conocimiento poderoso en el proceso de formación docente. Por lo tanto, existen importantes ventajas para los procesos de formación, que se evidencian por la polarización de los canales, generando importantes intercambios que los docentes pueden hacer, pero también limitando por la imposibilidad de experiencias reales y fácticas de los eventos de la profesión docente que deben figurar en los procesos formativos.

\section{REFERENCIAS}

BENTET GIL, A. et al. Nuevos horizontes formativos: una experiencia del MOOC como recurso en la formación continua. Apertura, v. 10, n. 1, p. 88-103, 2018.

CALVO SALVADOR, A.; BRAGA BLANCO, G. M. Abriendo la formación de los profesionales de la educación al campo de los mooc. Resultados de un proyecto de investigación en las universidades de cantabria y oviedo. Revista de curriculum y formación del profesorado, v. 23, n. 2, p. 259-276, 2019. 
GALAVIZ PEÑA, J. M. Análisis comparativo en el uso de las tics para aplicaciones educativas de la competencia tecnológica. TLATEMOANI Revista Académica de Investigación, v. 15, n. 2, p. 188-204, 2014.

GIL QUINTANA, J. MOOC “Innovación Educativa y Desarrollo Profesional. Posibilidades y Límites de las TIC". Una Experiencia desde la Educomunicación en el Proyecto Europeo ECO. Qualitative Research in Education, v. 4, n. 3, p. 299-328, 2015.

GIL QUINTANA, J. Interactividad y participación desde el modelo comunicativo de los MOOC. Index.comunicación, v. 7, n. 1, p. 269-288, 2017.

LACERDA RABELLO, C. R.; AMARAL TAVARES, K. C. Tecnologias digitais no ensino superior: implementação e avaliação de um curso on-line de formação docente. Revista Linguagem \& Ensino, v. 20, n. 2, p. 215-262, 2017.

MALLAMANN, E. M.; NOBRE FERREIRA, A. M. Um canal aberto no ensino superior? MOOC e REA no mundo digital. Apertura, v. 9, n. 2, p. 24-41, 2017.

MANOTAS SALCEDO, E.; PÉREZ RODRÍGUEZ, A.; CONTRERAS PULIDO, P. Análisis de Vídeo-lecciones en MOOC enfocados en la formación pedagógica de docentes en Educación Superior. Un estudio de caso. REIDOCREA, v. 7, p. 248-259, 2018.

MOREIRA TEIXEIRA, A. et al. MOOC “Competências digitais para professores": uma prática formativa inovadora. RIED. Revista Iberoamericana de Educación a Distancia, v. 21, n. 2, p. 243-261, 2018.

RODRÍGUEZ, A. J.; MOLERO, D. M. Conectivismo como gestión del conocimiento. Revista electrónica de Humanidades, Educación y Comunicación Social, v. 4, n. 6, p. 73-85, 2008.

ROMANOWSKI, J. P.; TEODORA ENS, R. As pesquisas denominadas do tipo "estado da arte" em educação Researches appointed as "state of art" in education. Dialogo Educ, v. 6, n. 19, p. 37-50, 2006.

VÁZQUEZ CANO, E.; LÓPEZ MENESES, E. Los mooc y la educación superior: la expansión del conocimiento. Revista de Currículum y Formación de Profesorado, v. 18, n. 1, p. 3-12, 2014.

\section{Notas} `La evolución del pizarrón a tiza en pizarras interactivas o digitales, demuestra los avances y la inclusión de la
tecnología en el aula. 